TAO, Vol. 8, No. 2, 189-202, June 1997

\title{
HF Doppler Backscatter Observations of the October 1995 Total Solar Eclipse
}

\author{
Dan Meehan $^{1}$
}

(Manuscript received 30 December 1996, in final form 9 April 1997)

\begin{abstract}
The Royal Australian Air Force operates an Over The Horizon Radar (OTHR) from Alice Springs in Central Australia. This OTHR is called Jindalee. Since the active medium for this form of radar is the ionosphere, Australia has a continuing interest in the physics of this region of the upper atmosphere.

One of the support instruments for Jindalee is a very much scaled down version of the radar, called the Mini-Radar. It forms part of the Frequency Management System (FMS), and it is used to provide operators of the main radar with advice on ionospheric conditions, and it operates in an identical fashion to the main radar, recording the signal strength of surface backscattered HF radio echoes as a function of range, Doppler and azimuth.

October 1995 marked the occurrence a total solar eclipse, in which the path of totality travelled eastward from near the Caspian Sea, past the Philippines and out into the Pacific Ocean. Since this region falls within the area "covered" by the Mini-Radar, it was decided to use this instrument to monitor ionospheric behaviour during the period of the eclipse.

Early results are presented.
\end{abstract}

\section{INTRODUCTION}

Australia's Jindalee OTHR is situated near Alice Springs in Central Australia [Pengelley 1988, Grutzner 1989]. It operates by transmitting HF radio waves at very low elevation angles up to the ionosphere, where they are refracted downwards and scattered from the Earth's surface in regions of interest. These radio waves are also scattered off any targets which happen to be within this area of interest. A very small fraction of the transmitted energy, returns to the receiver site where radar signal processing is used to identify any targets present.

An important part of running the radar is choosing optimal operating parameters such as radio frequency. To this end, there is a separate system called the Frequency Management System (FMS) which supplies real-time propagation advice to operators of the main radar

${ }^{1}$ Defence Science and Technology Organisation, Adelaide, Australia 
[Earl and Ward 1987].

One sub-system of the FMS is the Mini-Radar. This is a very much scaled down version of the main radar, with smaller transmitter and receiver arrays and smaller transmitted power. It is not used for target detections but serves purely as a support system, measuring various propagation conditions in 8 beam directions numbered from 0 to 7 , as indicated in Figure 1. The solid lines represent approximate beam edges and are numbered from 0 to 7, (west to north respectively). The elevation angles of the beams are approximately 15 degrees, though they do vary slightly with frequency.

The diurnal, seasonal and solar cycle variations in ionospheric conditions, specifically

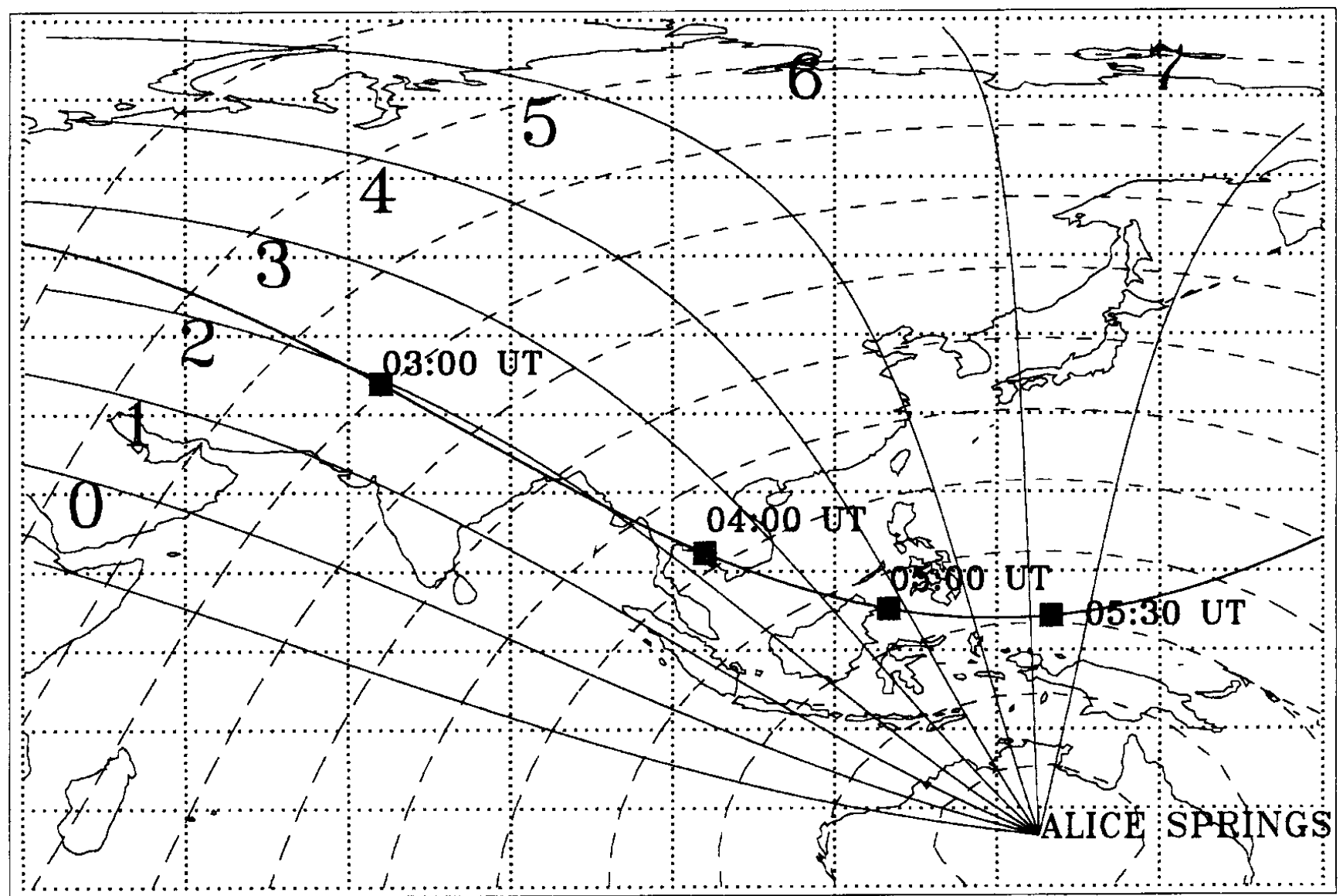

15 degree increments in Longitude, 10 degrees in Latitude

Fig. 1. Map of the Asian-Australian region with the approximate path of totality of the October 1995 Total Solar Eclipse. The solid lines radiating from Alice Springs represent the edges of the beams, the beams being numbered from 0 (to the west) through to 7 (to the north). The dashed arcs centred on Alice Springs show the distance from Alice Springs in 1000km increments.

backscatter, as perceived by Jindalee and the various support sub-systems, have been well documented in the last decade, [Ward and Elkins 1995]. Nonetheless, there always remains a need to test/refine our understanding of ionospheric dynamics. 
In October 1995, a total solar eclipse occurred and its path of totality travelled eastward through South East Asia. This fell within the "coverage" of the FMS support systems [See Figure 1], and therefore, it was decided to use the Mini-Radar to monitor ionospheric behaviour during the period of the eclipse.

A solar eclipse occurs when, for observers on Earth, the Sun's light is partially or totally blocked by the Moon. Every month (approximately), the Moon is between the Earth and the Sun, but the three bodies do not form a straight line and hence there is no eclipse. Even so, it is not totally rare that such "straight line geometry" does occur, meaning that partial eclipses are not terribly uncommon, averaging over 2 a year in the $21^{\text {st }}$ century [Meeus et al., 1966, and Ridpath 1990]. Of these, approximately $1 / 3^{\text {rd }}$ are total eclipses.

An important feature of the eclipse (for the current purpose) is the direction of travel of the region of totality. If a frame of reference is considered in which the Earth does not rotate then, just as the Sun appears to do each day, the Moon will rise in the east and set in the west. However, the Moon's speed across the sky is slower than that of the Sun. Thus, during an eclipse the shadow of the Moon on the Earth's surface travels towards the east, suggesting that any effects of an eclipse measured by the Mini Radar will appear first in the most westerly beams and progressively work towards the eastern beams.

Figure 1 is a map of the Australian/Asian region showing the path of totality of the eclipse of October 24, 1995. The markers show the position of totality as a function of time. In clear evidence is the eastward movement.

The eclipse should be expected to create a depression in the electron content in the various ionospheric layers directly within the shadow of the Moon [Ferraro 1963]. This is similar to the onset of evening when electron production decreases while recombination proceeds, resulting in a net loss of electrons. In the so-called D region of the ionosphere $(50-80 \mathrm{~km}$ altitude), this should produce a decrease in absorption and, therefore, an increase in echo strength from regions more distant than the eclipse path [Rawer 1956].

In the ionospheric $\mathrm{E}$ and $\mathrm{F}$ regions, this electron loss will manifest as a negative Doppler shift, with a positive shift occurring after the shadow has passed, due to the increase in the electron concentration as the sun once again illuminates the region [Ward and Elkins, 1995]. This alternating Doppler shift is readily observed every day with the mini-radar, owing to the passage of the dawn/dusk terminators, i.e. a negative shift at dusk and a positive shift at dawn.

Accordingly, the following predictions can be made:

(1) At ranges farther than the path of totality, there will be a negative Doppler shift in the radar echoes, followed by a positive shift, as the eclipse passes.

(2) The eclipse will allow propagation to increased distances, relative to distances recorded at similar times on days on either side of the day of the eclipse.

In the remainder of this report, whether or not these predictions have held true in the MiniRadar data is assessed.

\section{DATA}

Figure 2 shows the type of data collected by the Mini-Radar. It is for one time on the day of the eclipse. The Doppler shift (in $\mathrm{Hz}$ ) is shown on the $\mathrm{X}$ axis, the range being from $-5 \mathrm{~Hz}$ to 


\section{Beam 4 15MHz Day 45/1997 2:00UT}

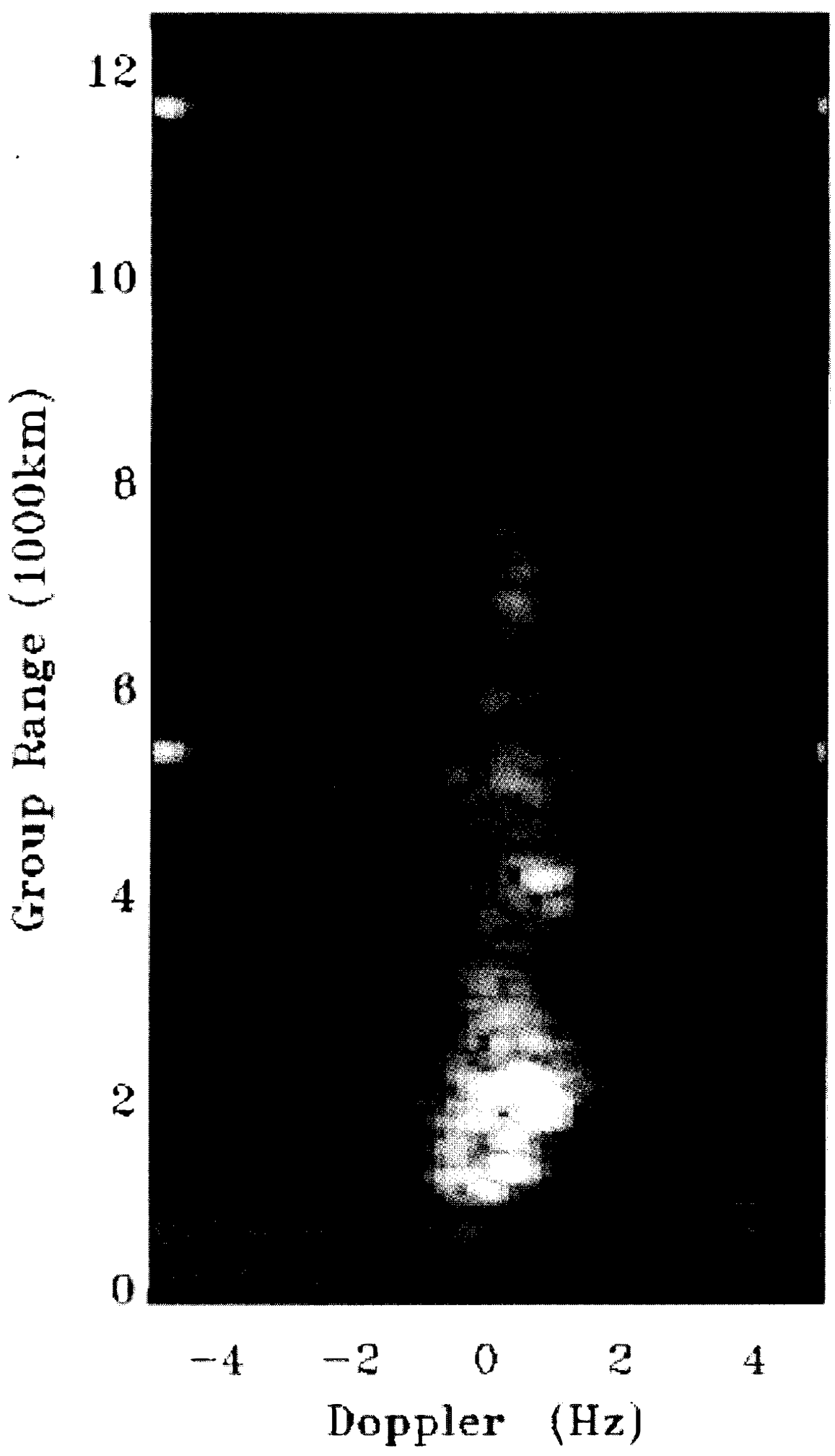

Fig. 2. Example of the type of data collected by the Mini-Radar. The echo signal strength is shown as a function of range and Doppler. The range is from $0 \mathrm{~km}$ to $12,500 \mathrm{~km}$, Doppler is $+/-5 \mathrm{~Hz}$, with $0 \mathrm{~Hz}$ in the centre. Signal power is color-coded rising from low values (black) to high values (white). 
$5 \mathrm{~Hz}$ with $\mathrm{OHz}$ in the centre. The Group Range is represented along the $\mathrm{Y}$ axis, and in this case extends from $0 \mathrm{~km}$ to $12,500 \mathrm{~km}$. This particular dwell of data is for beam 3, which points towards the north-west of Alice Springs. The data are colour coded for signal strength, in units of dBW/Doppler cell, with power increasing from black to white. The two dots on the left side of the Figure at ranges of approximately $5,000 \mathrm{~km}$ and $11,000 \mathrm{~km}$ are calibration signals, while the dark grey region at approximately $6,250 \mathrm{~km}$ is an artifact of the recording process. These data were recorded at $15 \mathrm{MHz}$.

All the data used in this report were recorded using a Linear Frequency Modulated Continuous Wave (FMCW) waveform with a waveform repetition frequency of $10 \mathrm{~Hz}$, and a sequence of radio frequencies starting at $21 \mathrm{MHz}$ and descending in $2.5 \mathrm{MHz}$ steps to the lower limit of $7.5 \mathrm{MHz}$. The most westward beams were not recorded as this would have reduced the amount of data from the other beams. The eclipse was expected to have had very little effect on these other beams because the trajectory meant that the point of totality was never in the direction of beams 0,1 or 2. For completeness, it would have been desirable to have data for these beams, but the decision had been made to focus on those beams which included the path of totality.

Figure 3 shows a summary of the $15 \mathrm{MHz}$ beam 3 data for three days centered on day 297 (the day of the eclipse). The many small panels are each the same type of data as in Figure 2, i.e. many small range/Doppler plots. Universal Time is along the overall $\mathrm{X}$ axis; the time stamps are shown above day 298 data. Individual panels are separated along the time axis by 20 minutes. For the direction of beam 3, consideration of Figure 1 suggests that the effects of the eclipse might have begun at around 3:00 UT to 4:00 UT. Figure 3 shows that for day 297 there was a "wobble" in Doppler at a range between $5,000 \mathrm{~km}$ and $7,000 \mathrm{~km}$ from approximately 03:30 to 05:30 UT, while this was absent on the days before and after 297 .

This "wobble" is in the predicted sense, i.e. a negative Doppler shift as the eclipse approaches, followed by a positive shift as electrons once again begin to grow in concentration.

Figure 4 presents another perspective from which to view the data. Figure 4(a) shows a Doppler frequency shift (in $\mathrm{Hz}$ ) at a fixed range and frequency, as a function of time and the Mini-Radar beam for day 296 (the day before the eclipse). Local time is UT $+9: 30$, which means that sunset was at approximately 8:00 UT. Sunset caused the ionosphere to rise and the accompanying decreasing electron content caused large negative Doppler shifts on the backscattered returns. Negative shifts can be seen in beam 7 at approximately 07:00. The negative shifts moved to lower beam numbers with time. Lower beam numbers imply more westerly directions, and so this pattern is consistent with sunset travelling to the west.

After sunset, the ionosphere often descended to the altitude which it would retain for most of the evening. This caused a positive Doppler shift, and this pattern can be seen to have been moving to the west an hour or so after the negative shift sequence. There is little indication of a pattern of Doppler shift swings in the period from 03:00 to 06:00 UT, the time when the eclipse existed on day 297.

Figure 4(b) shows similar data to those in 4(a), but for the day after the eclipse. The data is unfortunately truncated in time due to equipment problems. Nonetheless, the negative shifts can be seen moving down in beam numbers, (travelling towards the west). Again there was no pattern of shifts in the period 03:00 - 06:00 UT. 
1995 Eelipse Data Beam $3 \quad 15 \mathrm{MHz}$

OUT $2 \mathrm{Cr}$

3UT

+ UT

$5 \mathrm{UT}$

a or

$\%$ UT

$80 T$
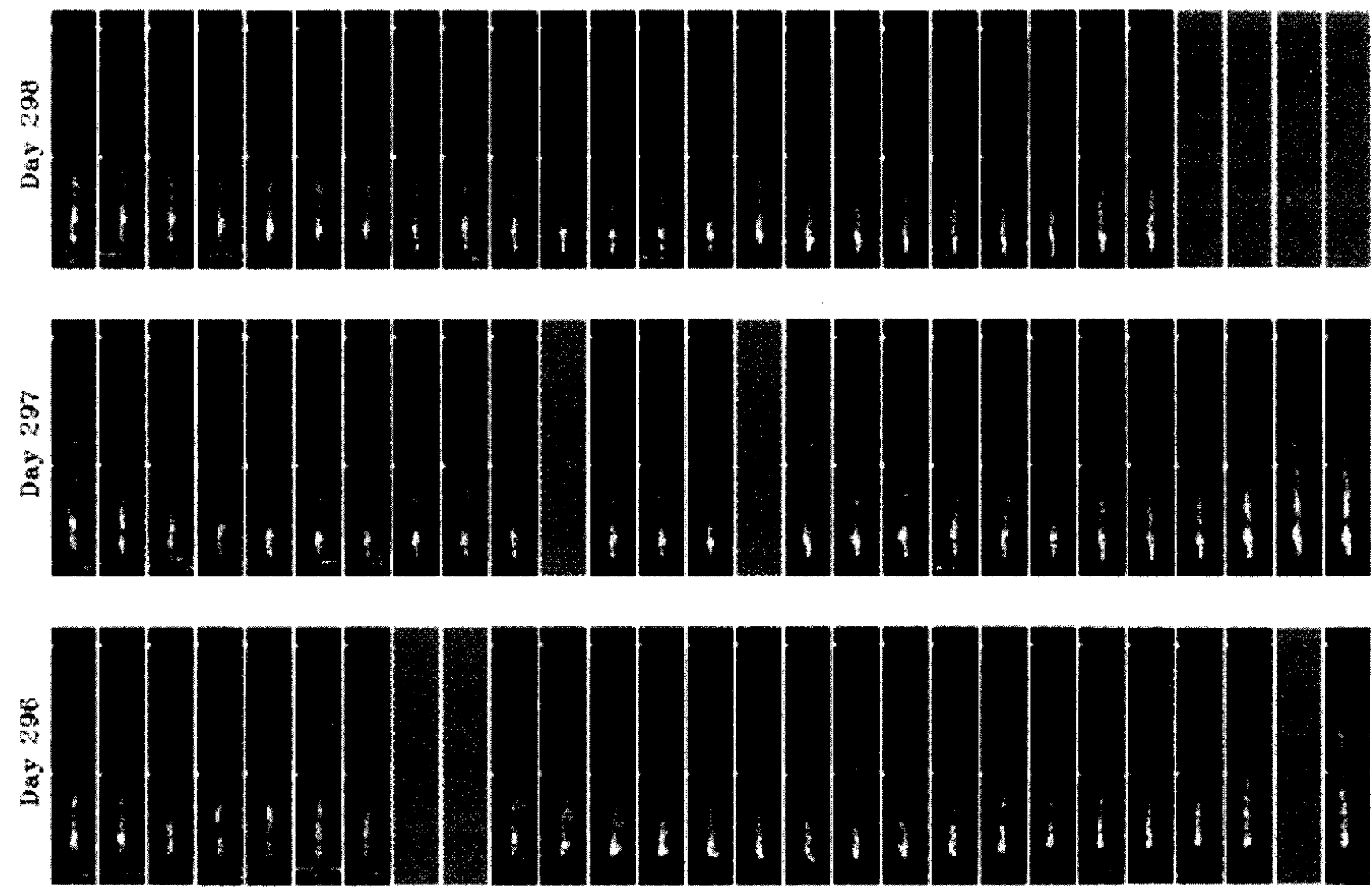

Fig. 3. Three time sequences of mini-radar data are shown: for the day before the eclipse (296), the day of the eclipse (297), and the day after the eclipse (298) as indicated. Each small panel shows data in a similar form to those in Figure 2. The effects of the eclipse can be seen on the sequence of day 297 at approximately 03:40 UT up until approximately 06:20. For example, at a range of $5,000 \mathrm{~km}$, the echoes move first to the "left" (negative Doppler), and then to the "right" (positive Doppler). Also, throughout this period, there is an increased distance of propagation compared to similar periods on days 296 and 298, although this effect is less obvious than that for the Doppler shift.

Now Figure 4(c) represents data for day 297, the day of the eclipse. The sunset pattern was evident once more; however this time there was a pattern of Doppler shifts which began in beam 3 and moved to higher beam numbers. The first movement was negative shifts, but was then followed by a positive Doppler. This pattern was in the period 03:00 to 06:00 UT, showed the predicted negative/positive sequence and travelled in the opposite direction to the other Doppler patterns we have seen.

To examine the expected enhancement of long range propagation during the eclipse, it is important consider Figure 3. On the day of the eclipse, starting at approximately 03:00, there was much more energy returning from ranges greater than $6,000 \mathrm{~km}$, than on the days on either 


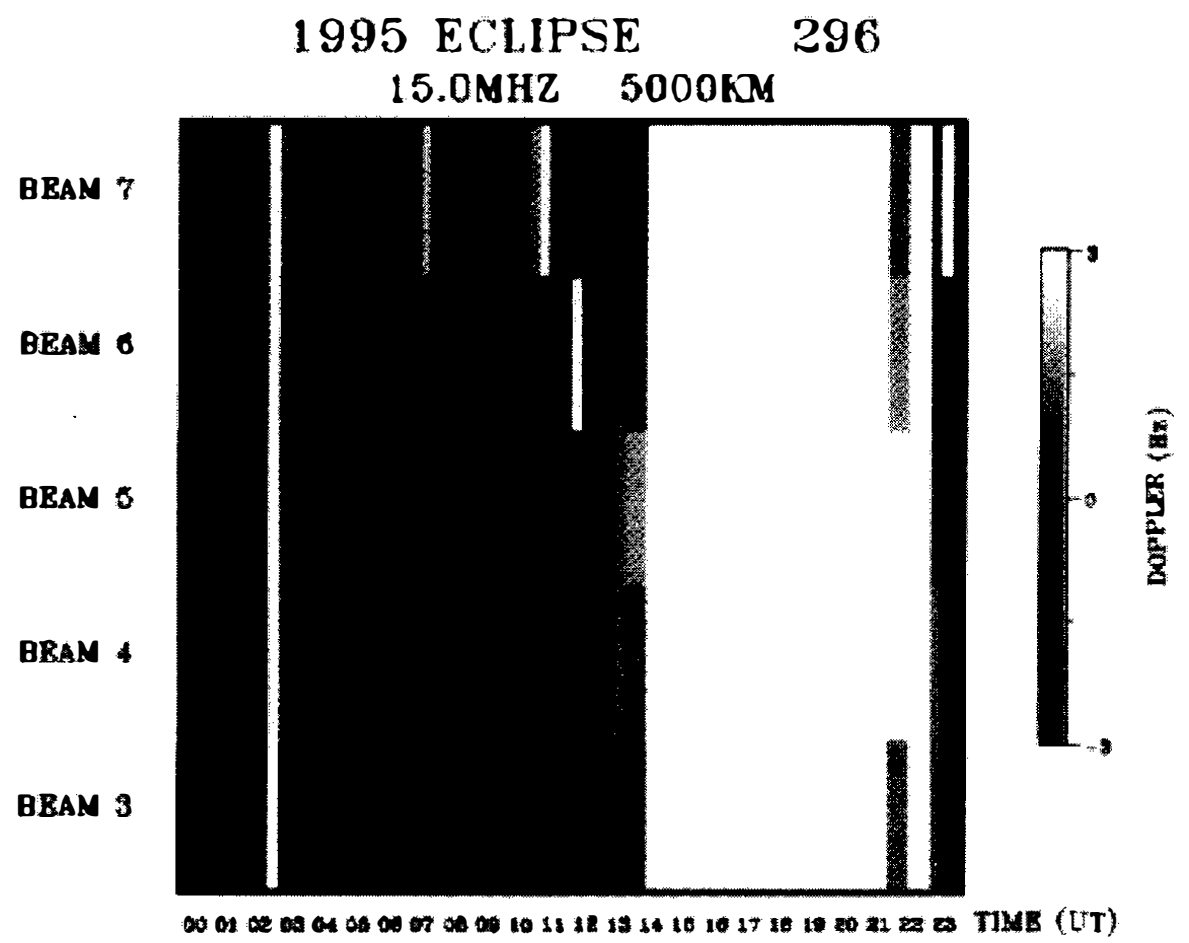

Fig. 4(a). Doppler shift as a function of time and beam for the day before the eclipse. The negative Doppler shifts due to the effects of sunset can be seen starting in beam 7 (the most easterly beam) at 06:00 UT, progressing with time to beam 3 at 10:00 UT.

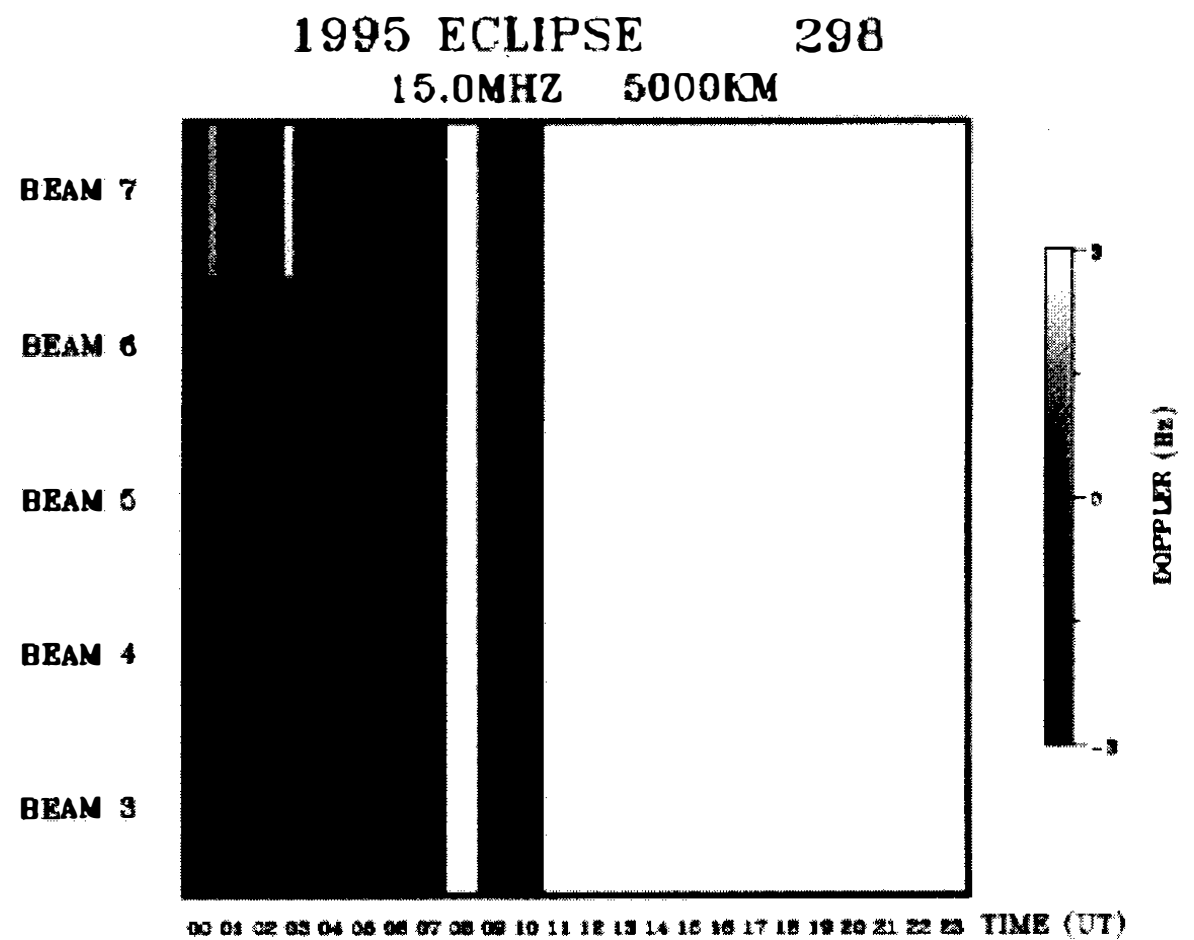

Fig. 4(b). Doppler shift as a function of time and beam for the day after the eclipse. Once more, the negative Doppler shifts due to the effects of sunset can be seen starting in beam 7 progressing through to beam 3 . 


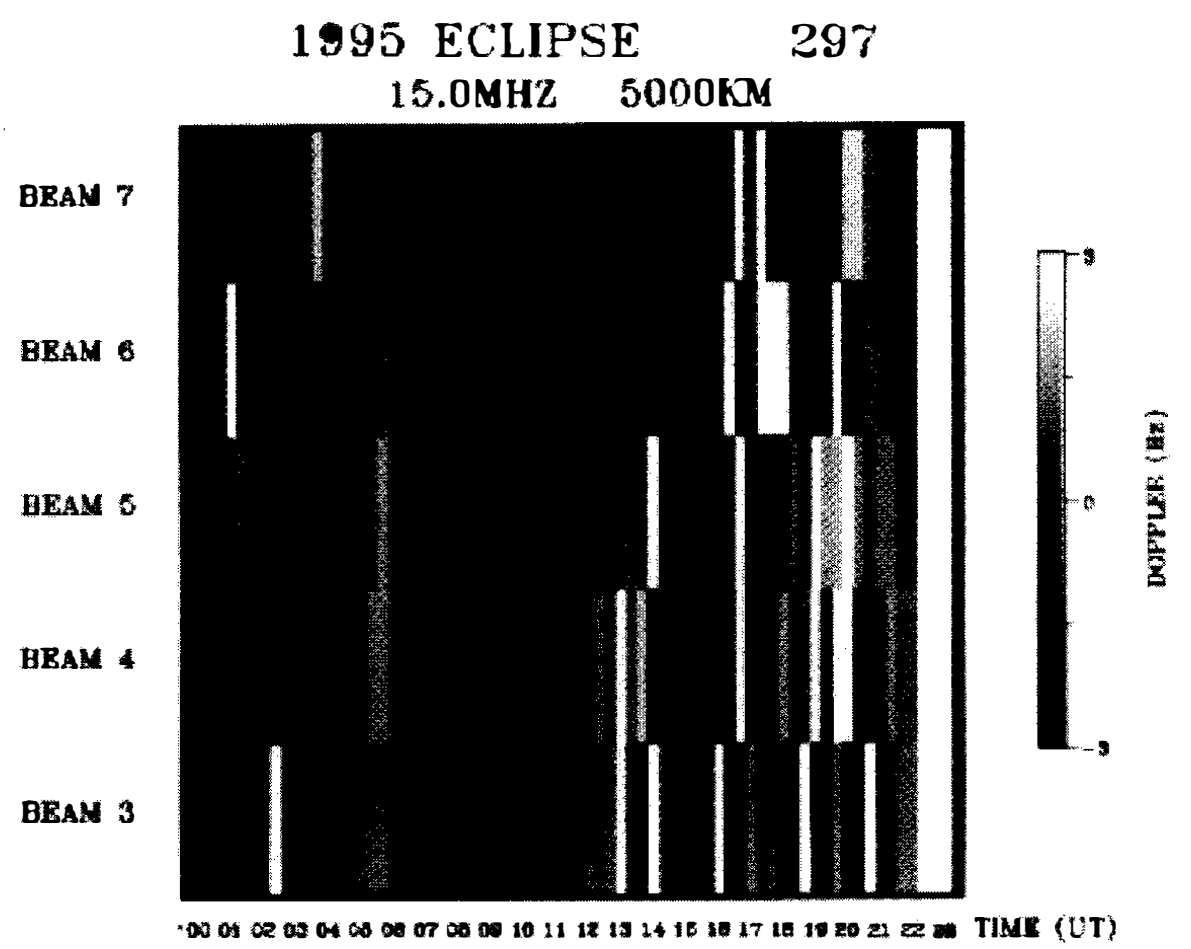

Fig. 4(c). Doppler shift as a function of time and beam for the day of the eclipse. Again, the negative Doppler shifts due to the effects of sunset can be seen starting in beam 7 progressing with time to beam 3 . However, in this diagram of note is the extra feature of the negative and positive Doppler shift "wobbles" tracking from beam 3 first through to beam 7 at a later time. This is suggestive of a movement toward the east, exactly as expected of a solar eclipse.

side. Figure 5 shows signal power data in dBW in a similar format to those in Figure 4 (a). Figure 5(a) shows data for the day before the eclipse, and at times near 04:00; no clear pattern of clutter power was observed. Sunset produced strong power returns and once again this pattern could be seen starting in beam 7 at around 08:00 UT and then migrating to the more westerly beams.

Figure 5(b) shows data for the day of the eclipse, and here what is seen is that the eclipse pattern of stronger clutter returns beginning in beam 3 and moving with time to beam 7 though the pattern is far less clear than that for Doppler shift. Figure 5(c) shows data for the day after the eclipse and once again there was no obvious pattern which might have coincided with the period of the eclipse.

Both the Doppler shift and clutter power data follow the predicted beam/time patterns, which seems to be strong support for our having observed the passage of the eclipse.

It is important that when trying to observe the effects of some physical event, other conditions remain constant. Appendix A shows information from the Australian IPS geophysical summary for days surrounding the eclipse. Apart from the eclipse, there was little other activity which might have disturbed the ionosphere. 


\section{ECLIPSE 296}

15.0MHZ 7000KM

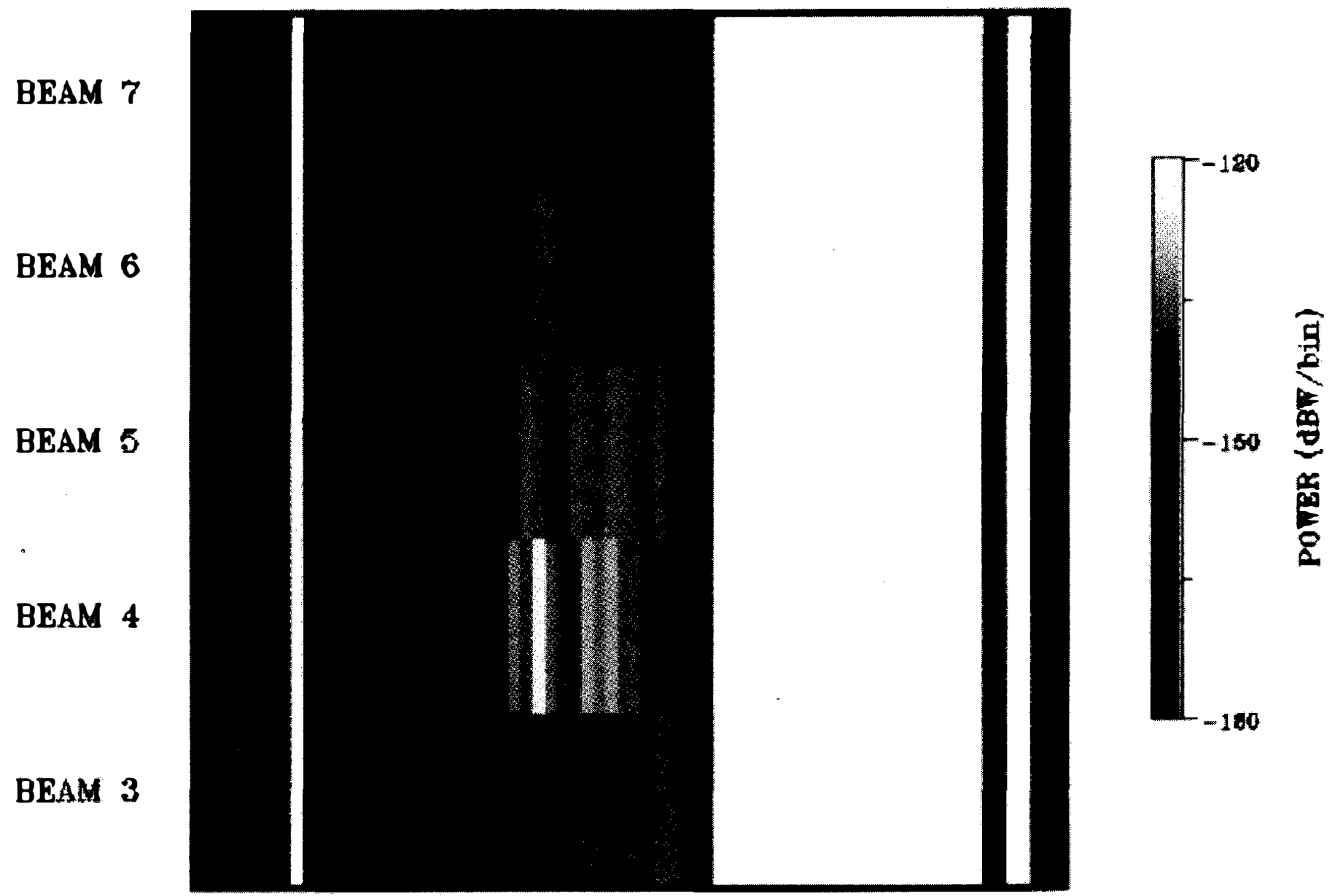

0001020304060607080910111213141616171019202122 2s TIME (UT)

Fig. 5(a). Echo power as a function of time and beam for the day before the eclipse. During the period of 03:30 to 06:30, there is no obvious "signature" of an unusual event. Sunset is evident through the increased echo powers from approximately 07:00 UT onward.

\section{DISCUSSION}

Under examination now are the relative roles of the umbra and penumbra in causing the effects noted here.

Figure 6 shows a sequence of geographical maps of the region to the north-west of Australia. Superimposed on each small map is a line showing the path of the umbra. A black and white rectangular marker shows the position of the umbra (at ground level) with time. The quantity graphed is once again the Doppler shift.

It is worth noting that the commencement of negative Doppler shifts seems to precede the arrival of the umbra. For example, in the plot second from the bottom and immediately after the blank space, the more easterly beams show negative shifts while the point of totality has 


\section{ECLIPSE 297 \\ 15.0MHZ 7000KM}

BEAM 7

BEAM 6

BEAM 5

BEAM 4

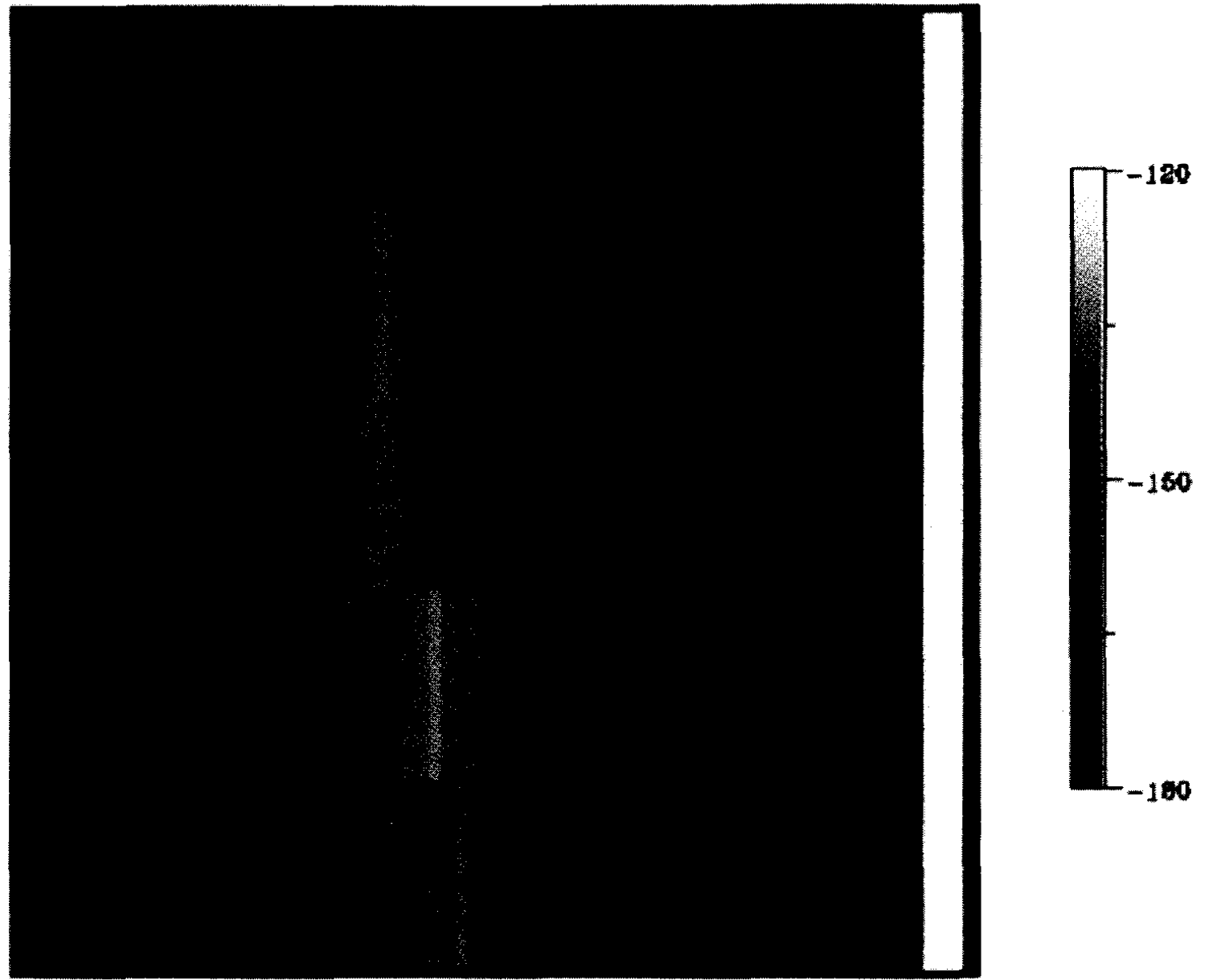

BEAM 3

000102050406000708001011121514161617101920212220 TIME (UT)

Fig. 5(b). Echo power as a function of time and beam for the day of the eclipse. In beam 4 at 05:00 UT, there is an increase in echo strength which tracks into beam 7 with time. This is precisely what is expected. Later in the day, the effects of sunset moving from beam 7 through to beam 3 are observed.

not reached these beams. A very approximate estimate puts this "lead" distance at possibly $2,000 \mathrm{~km}$, (with a very large error bound). Equally, there is a suggestion of negative shifts at ranges smaller than that of the point of totality.

It must considered that the positions of the eclipse path are given for ground level. The position of the umbra at certain ionospheric heights, say $200 \mathrm{~km}$, is skewed towards the Sun, and at sunrise the sideways displacement can be very large indeed. However, the first part of this eclipse occurs at approximately 08:30 local time (Afghanistan), and the eastward displacement in position is of the order of $400 \mathrm{~km}$. This displacement with altitude decreases as the local time approaches noon, and then increases through the afternoon. The eclipse ceases after 05:30 UT, when the difference in ground and ionospheric position is estimated at less 


\section{ECLIPSE 298}

15.0MHZ $7000 \mathrm{KM}$

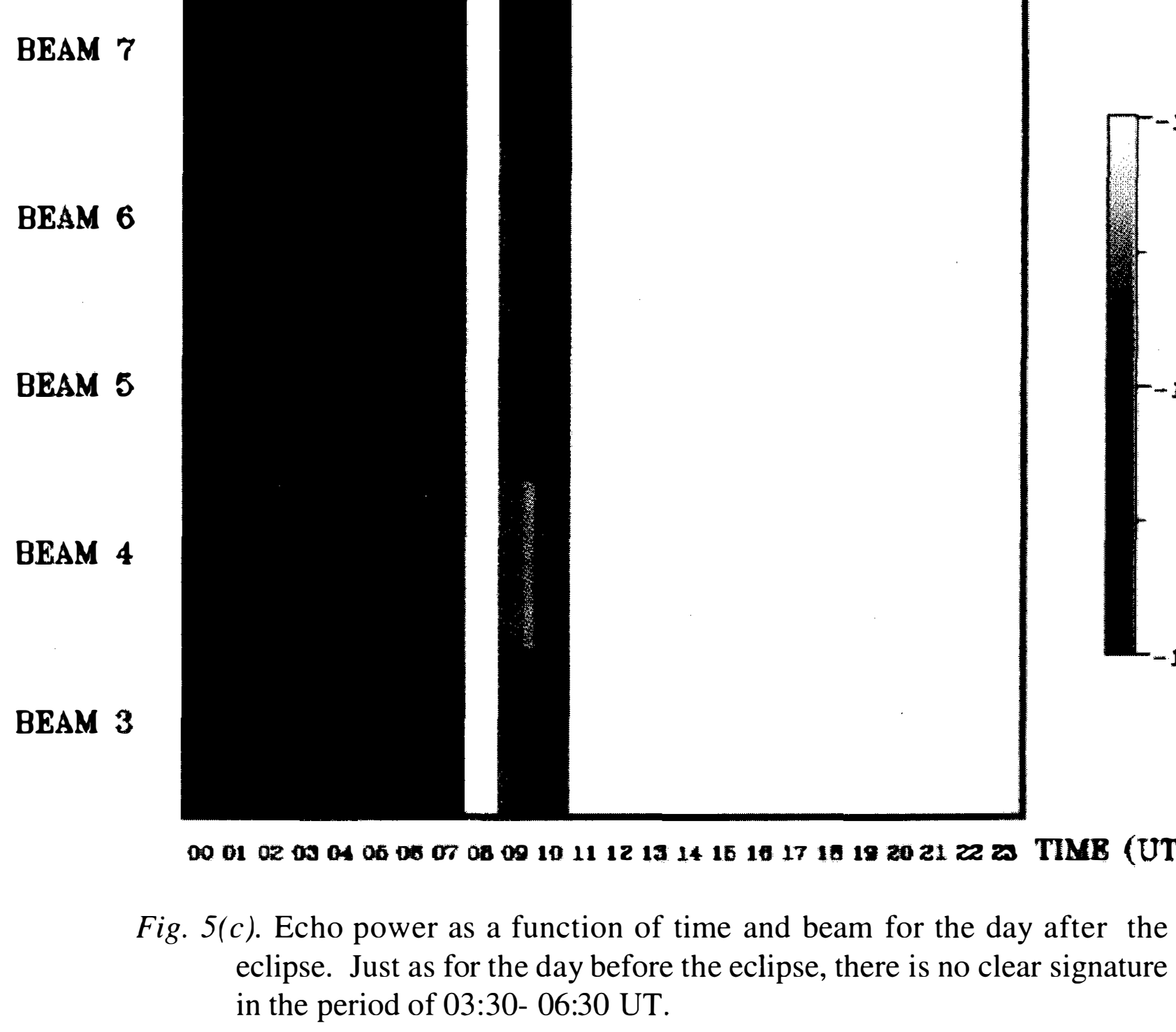

than $100 \mathrm{~km}$. On this basis, the commencement of the observed effects $2,000 \mathrm{~km}$ before the arrival of totality and also at ranges closer than the path of totality, are not caused by the difference in positions of the eclipse at ground level and at ionospheric heights.

This strongly suggests that the grey shadow of the Moon (the partial eclipse region or penumbra) plays some role in bringing about the effects measured by the Mini-Radar. This is perhaps not surprising given that the penumbra is on average 400 times the area of the umbra [Baker 1961].

As the point of totality moves out of the coverage of the Mini-Radar, the positive Doppler shifts can be seen, as expected.

The grey areas on the plots in Figure 6 show the night-time regions of the Earth, and in the last two maps of this Figure, the negative Doppler shifts associated with sunset can be observed. 

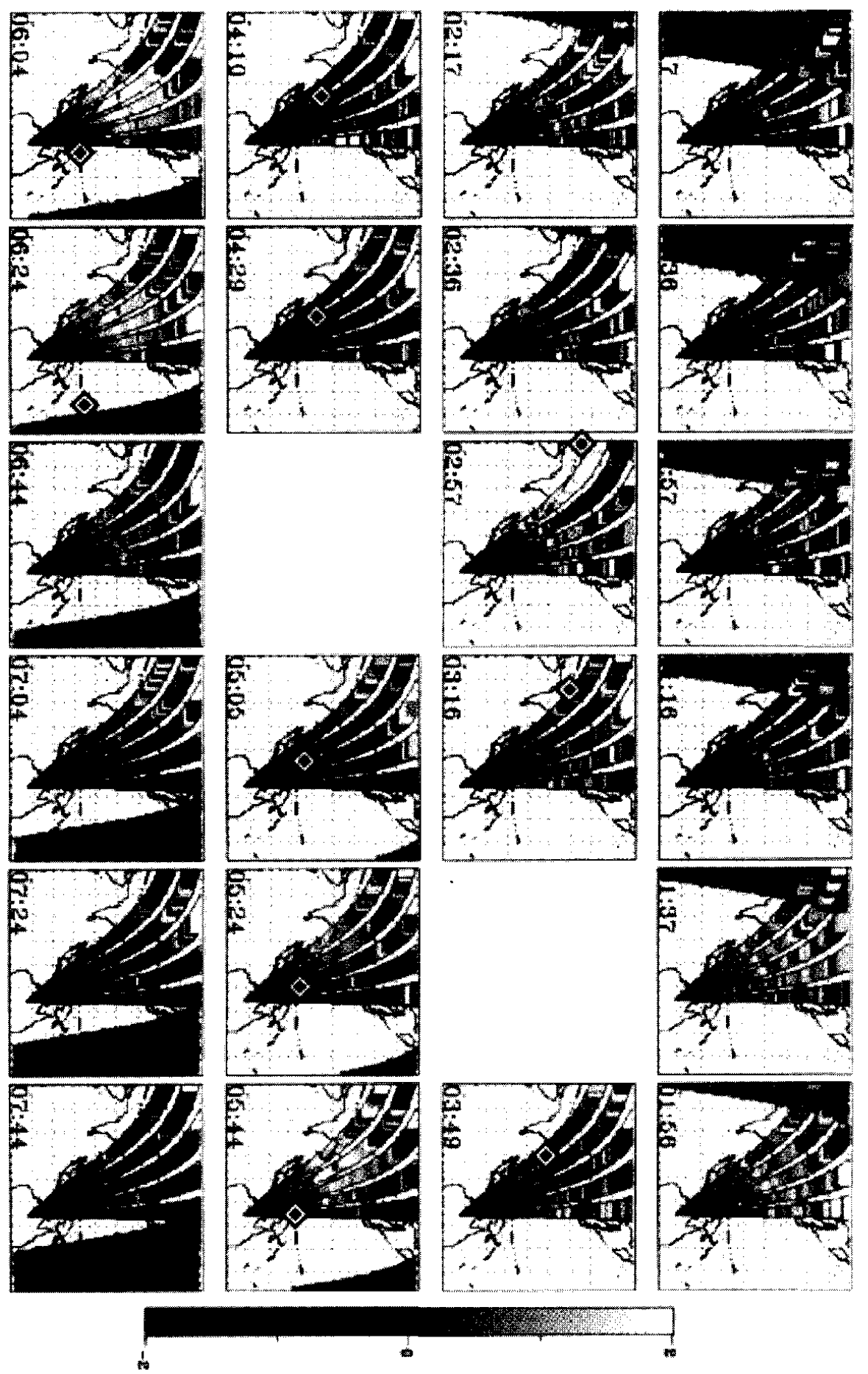

Doppler tr

Fig. 6. A time series of the Doppler shift versus range and azimuth, superimposed on a map of the Asia-Australian region is presented. The interval between maps is 20 minutes, and the dashed line shows the approximate path of totality. The rectangle showing the position of totality is first seen in the panel fourth in from the left and second from the top (03:16 UT), while it is last seen in the 06:24 UT panel. Of interest is the fact that the Doppler shifts are negative "in front of" totality, and positive "behind" it. 


\section{CONCLUSIONS}

It seems clear that it has been possible to monitor the passage of the solar eclipse of October 1995 at a distance of several thousand kilometres.

The behaviour of the ionosphere is in qualitative agreement with the theory, and the penumbra seems to be involved in producing effects which are undoubtedly due to the vastness of the area when compared to the size of the umbra.

Ray tracing studies will be conducted in the future to quantitatively test the current understanding of these events and of the ionosphere. As well, the data will be investigated further to determine whether the eclipse triggered travelling ionospheric disturbances as discussed by Singh et al 1989.

It is hoped that observations will have been be made of the March 1997 total solar eclipse, which passed much farther to the north than the coverage focussed on in this report.

\section{REFERENCES}

Baker, R.H., 1961: An Introduction to Astronomy. $6^{\text {th }}$ Edition, D. Van Nostrand Company, Princeton, New Jersey.

Earl, G.F. and B.D. Ward, 1987: The frequency management system of the Jindalee over-thehorizon backscatter HF radar. Radio Sci., 22, 275-291.

Ferraro, VCA, J.E.C. Gliddon and P.C. Kendall,1963: Solar Eclipse and solar flare effects in the F2 region. Proceedings of the international conference on the ionosphere 1962. Published by The Institute of Physics and the Physical Society, London, .

Grutzner, A., 1989: Radar Love. Somewhere, over the horizon. Electronics Today International, 45-48.

Meeus, J., C.C. Grosjean and W. Vanderleen, 1966: Canon of Solar Eclipses. Pergamon Press, Oxford.

Pengelley, R., 1988: Australia's all-purpose early warner. International Defense Review, 3, 285-288.

Rawer, K., 1956: Absorption Measurements for the Eclipse of 30 June 1954. In :W.J.G. Beynon and G.M. Brown (Eds.),Special Supplements to Journal of Atmospheric and Terrestrial Physics, Solar Eclipses and the Ionosphere. vol. 6, Pergamon Press, London.

Ridpath, I., 1990: Norton's 2000.0. 18 $8^{\text {th }}$ Edition. Longman Scientific and Technical, (co-published with Wiley and Sons, New York), .

Singh, L., R.T. Tuhi, Y.V. Somayajulu, P.N. Vijayakumar, R.S. Dabas, B. Loganadham, S . Ramakrishna, P.V.S. Rama Rao, A. Dasgupta, G. Navneeth, J.A. Klobuchar, G.K. Hartmann, 1989: A multi-station radio beacon study of ionospheric variations during total solar eclipses. J. Atmos. Terr. Phys., 51, 271-278.

Ward, B.D. and Elkins, T., 1995: OTHR Radar Clutter - Observations and Characterisation. A report for the defence science and technology organisation of Australia. DSTO-RR0058, Published by The Defence Science and Technology Organisation of Australia, Adelaide, Australia, Jointly Published in the USA by Air Force Materiel Command as Report ESC-TR-95-103. 


\section{APPENDIX A}

The following presents the author's summary of the IPS WEEKLY SOLAR AND GEOPHYSICAL REPORT ISSUED ON 27 OCTOBER 1995, BY IPS RADIO AND SPACE SERVICES, FROM THE REGIONAL WARNING CENTER (RWC), SYDNEY, AUSTRALIA.

\section{SUMMARY ( 20 OCTOBER - 26 OCTOBER )}

$\begin{array}{llllllll}\text { Date } & 20 & 21 & 22 & 23 & 24 & 25 & 26 \\ 10 \mathrm{~cm} & 83 & 81 & 80 & 77 & 74 & 74 & 74 \\ \mathrm{~A} & 23 & 12 & 10 & 20 & 9 & 5 & (4) \\ \mathrm{T} & -11 & -13 & 6 & 12 & -4 & 5 & 2 \\ \text { Solar* } & \mathrm{VL} & \mathrm{VL} & \mathrm{VL} & \mathrm{Vl} & \mathrm{VL} & \mathrm{Vl} & \mathrm{VL} \\ \text { Magneic** } & \mathrm{A} / \mathrm{MS} & \mathrm{QU} & \mathrm{QU} & \mathrm{QU} & \mathrm{QU} & \mathrm{Q} & \mathrm{Q}\end{array}$

* VL means Very Low Activity

** A/MS means Active to Minor Storm levels. QU means Quiet to Unsettled, and Q means Quiet.

For the whole period, frequencies were generally near the predicted monthly values. 